\title{
Head and neck cancer: improving outcomes with a multidisciplinary approach
}

This article was published in the following Dove Press journal:

Cancer Management and Research

18 August 2017

Number of times this article has been viewed

\author{
Cristiana Lo Nigro' \\ Nerina Denaro' \\ Anna Merlotti ${ }^{2}$ \\ Marco Merlano' \\ 'Department of Oncology, \\ 2Department of Radiation Oncology, \\ S. Croce and Carle Teaching Hospital, \\ Cuneo, Italy
}

\begin{abstract}
For early-stage head and neck cancer (HNC), surgery (S) or radiotherapy (RT) is a standard treatment. The multidisciplinary approach, which includes multimodality treatment with S followed by RT, with or without chemotherapy (CT) or concurrent chemoradiotherapy (CRT), is required for locally advanced head and neck cancer (LAHNC). CRT improves prognosis, locoregional control (LRC), and organ function in LAHNC, compared to RT alone. Prognosis in recurrent/metastatic HNC (R/M HNC) is dismal. Platinum-based CT, combined with the anti-Epidermal Growth Factor Receptor (EGFR) antibody (Ab) cetuximab, is used in first-line setting, while no further validated options are available at progression. The complexity of disease is, in part, due to the heterogeneity of organs and functions involved and the need for a multimodality approach. In addition, the patient population (often elderly and/or patients with smoking and alcohol habits) argues for an individually tailored treatment plan. Furthermore, treatment goals - which include cure, organ, and function preservation, quality of life and palliation - must also be considered. Thus, optimal management of patients with HNC should involve a range of healthcare professionals with relevant expertise. The purpose of the present review is to 1) highlight the importance and necessity of the multidisciplinary approach in the treatment of $\mathrm{HNC}$; 2) update the knowledge regarding modern surgical techniques, new medical and RT treatment approaches, and their combination; 3 ) identify the treatment scenario for LAHNC and R/M HNC; and 4) discuss the current role of immunotherapy in HNC.
\end{abstract}

Keywords: HNC, multimodality treatment, multidisciplinary team

\section{Introduction}

Head and neck squamous cell carcinoma (HNSCC) is a heterogeneous disease, encompassing a variety of tumors that originate in the hypopharynx, oropharynx, lip, oral cavity, nasopharynx, or larynx. The disease group as a whole is associated with different epidemiology, etiology, and therapy. Worldwide, it represents the sixth most common neoplasia and accounts for $6 \%$ of all cases, being responsible approximately for $1 \%-2 \%$ of tumor deaths. ${ }^{1}$ Given the complexities of head and neck cancer (HNC), treatment decisions have to be taken by multidisciplinary teams (MDTs) with training not only in treatment but also in supportive care (considering swallowing, nutritional, dental, and voice impairment due to the effects of clinical intervention).

Tobacco and alcohol use has been associated with HNSCC. Infection with high-risk human papillomaviruses (HPVs), especially type 16, has been more recently implicated
Correspondence: Cristiana Lo Nigro Oncology Department, S. Croce and Carle Teaching Hospital, via Carle 25, I2100 Cuneo, Italy

Tel +390171616338

Fax +39 017I 61633I

Email lonigro.c@ospedale.cuneo.it 
in the pathogenesis of HNSCCs arising from the oropharynx. Given the more favorable prognosis, HPV-associated oropharyngeal cancer (OPC) represents a distinct clinical and biological tumor. ${ }^{2,3}$ Patients with HPV-driven diseases are younger, with less comorbidities and the disease is more chemo and radiosensitive. Trials are ongoing to establish if patients with HPV-driven disease should be treated with lessintensive therapy. ${ }^{4}$

Local therapy is effective on $60 \%-95 \%$ of patients with early-stage disease (both HPV- and environment/lifestyledriven). Survival and cure importantly benefit from early diagnosis and appropriate treatment. Both surgery $(\mathrm{S})$ and radiotherapy (RT) alone achieve satisfactory outcomes. ${ }^{1}$

The majority of HNSCC patients present with stage III and IV (locally advanced head and neck cancer [LAHNC]). Patients with LAHNC require multimodality treatment. In this setting, chemoradiotherapy (CRT) is the standard approach, ${ }^{5}$ although, in some patients (with bulky disease where organ preservation strategies are appropriate), induction chemotherapy, followed by cetuximab-RT (bio-RT) or CRT or S, may be used. ${ }^{6}$ Moreover, bio-RT may be an alternative for patients not fit to undergo cisplatin-RT. ${ }^{7}$

The disease control rate for LAHNC is about $40 \%$ at 5 years; acute and late toxicities remain a challenge. Recent data focus on the role of supportive care in reducing acute and late toxicities; early evaluation of pretreatment conditions, swallowing impairment, and new side-effect onset improves outcomes and quality of life (QoL). ${ }^{8}$ For recurrent/metastatic $(\mathrm{R} / \mathrm{M})$ disease, CT remains the standard therapeutic option. After platinum progression, no second lines that significantly improve prognosis are available. ${ }^{1}$

For this reason, molecularly targeted drugs, and recently immunotherapy, have become very important to improve outcomes, and their clinical studies are ongoing. While unsatisfactory results were obtained by standard target therapy, promising clinical data have come from immunotherapy. ${ }^{9}$ In fact, emerging data underlined a major role of the immune system in tumor development and progression, suggesting a key prognostic value in HNSCC. ${ }^{10}$

In the past, surgery for OPC was mainly performed through transfacial incisions so that many patients required extensive adjuvant postoperatively CRT. MDTs aimed to identify alternatives, such as transoral endoscopic head and neck surgery (eHNS) and transoral robotic surgery (TORS), in order to save function and cosmesis. These options have subsequently emerged as a key, minimally invasive, part of multidisciplinary care for HNC. ${ }^{11}$

\section{Importance and necessity of the multidisciplinary approach in the treatment of HNC}

HNC treatment is intrinsically complex. Nutritional and swallowing evaluation, dentary preparation, and pain management are mandatory before, during, and after concomitant treatment. $^{12-15}$

Therefore, an MDT should include not only an ear, nose, throat surgeon, radiation oncologist and medical oncologist, and radiologist but also a dietician, dentist, pain physician, and swallowing physician.

To apply the multidisciplinary approach in LAHNC, patients should be referred to a tertiary center when the MDT is not available. Conducting regular MDT meetings requires time and financial investment.

Pillay et $\mathrm{al}^{16}$ reviewed 72 articles analyzing the impact of MDT decisions on cancer patients: there was limited evidence for improved overall survival (OS) in patients followed by MDTs. Changes in diagnostic procedures were reported in $4 \%-45 \%$ of patients discussed at MDT meetings, who, likely, received more accurate preoperative staging and neoadjuvant/ adjuvant therapy. ${ }^{16}$

In the Australian retrospective analysis by Kelly et al, ${ }^{17}$ patients post-MDT (vs those not followed by MDT) had higher rates of dental evaluation ( $59 \%$ vs $22 \%, p<0.0001$ ), nutritional evaluation ( $57 \%$ vs 39\%, $p=0.015$ ), PET staging ( $41 \%$ vs $2 \%$, $p<0.0001)$, CRT for LAHNC (66\% vs 16\%, $p<0.0001)$, and adjuvant-CRT for high-risk patients ( $49 \%$ vs $16 \%, p<0.0001)$. In the MDT group, the time between S and RT ( $p=0.009)$ and the length of hospitalization $(p=0.002)$ were shorter. ${ }^{17}$

Italian data on 781 patients from the Milan Institute indicated higher requests for new staging ( $49 \%$ of patients) and modification in therapeutic program (10\% of patients) following MDT assessment. Thus, in a tertiary referral hospital, an MDT approach ends to staging refinement or therapeutic changes in about $60 \%$ of patients. ${ }^{18}$

MDT approach positively affects survival, as shown in studies from South and West Audit of Head and Neck Cancer I and II and Cincinnati Veteran's Administration Hospital (Australian study and Chinese study). ${ }^{19-22}$

An important role of multidisciplinary management should involve consideration of costs, available infrastructure treatments (including preparation and early management of known acute toxicities), and QoL issues. Moreover, in clinical practice, an inadequately set-up MDT is less likely to impact positively on decisions and outcomes for $\mathrm{HNC}$ 
patients. Given the complexities of treatment, the rationale for the use of an MDT to define individual optimal treatment strategies on a per-patient basis is apparent. Some data did not confirm the advantage of more speedy diagnosis and treatment, which could have been a reflection on a lack of clear roles and responsibilities of team members. Finally, expert practical advice on the implementation of MDTs may enable the establishment of this approach more universally around the world. ${ }^{23}$

In the guidelines issued by the National Comprehensive Cancer Network, HNC patients are recommended to receive treatment at centers with expertise. ${ }^{1}$ An American multicenter study confirmed an impact on survival ${ }^{24}$ with such an approach. Moreover, patients treated at institutions with high clinical trial accrual volume (compared with centers with low accrual) experienced lower RT protocol deviations (6\% vs $18 \% ; p<0.001)$ and higher survival (5 years: $69.1 \%$ vs $51.0 \% ; p=0.002$ ). Treatment in less experienced centers was referred to a higher death risk of $91 \%$ (hazard ratio [HR] $=1.91 ; 95 \%$ CI, 1.37 to 2.65$).{ }^{24}$ Table 1 summarizes data on multidisciplinary approach in HNSCC.

\section{Updated knowledge on modern surgical techniques, new medical and RT treatment approach, and their combination}

\section{Surgery}

Transoral eHNS either using laser or robotic methodologies has emerged as a new approach for the surgical management of OPC. There are clear advantages to eHNS - such as the lack of external incisions and significant ease of access over more conventional methods. The oral route provides a means for gaining access using robotic/laser techniques. Furthermore, this facilitates good visualization of oropharyngeal tumors and results in less scarring and disfigurement, with a significant reduction in speech and swallowing impairment for the patient. The benefits from employing robotic and laser approaches are manifold and in some ways mirror the advances made using intensity-modulated RT approaches comparing with $2 \mathrm{D}$ and $3 \mathrm{D}$ conformal techniques. The current literature describing prospective clinical trials is supportive of the approach of RT plus or minus chemotherapy for the management of OPC. However, there is currently a paucity of published data describing the more recent approach of transoral endoscopic surgery for this disease. ${ }^{11}$

Several trials are ongoing and preliminary reports were recently presented, focusing on a new role for surgery in organ/function preservation.

Transoral eHNS is minimally invasive surgery of the OPC, performed with either transoral laser microsurgery $(\mathrm{TLM})^{25}$ and/or TORS. ${ }^{26}$ Both techniques provide a highly magnified image of the tumor, allowing precise resection of various cancer invaginations, usually not visualized with standard surgery.

TORS has been used for the pharyngeal and laryngeal cancer resection, with the aim of improving function and esthetics, without worsening OS.

This innovative surgical technology allows for

- improved visualization;

- enhanced surgical safety (image guidance with CT scan and/or MRI);

- improved reach (using flexible instrument arms);

- real-time histopathological information and rapid informatic elaboration;

- seated surgery (that permits a true $3 \mathrm{D}$ visualization of hypopharynx);

- remote telementoring.

In large and complex OPC, TORS can resect lesions, avoiding a lip-splitting approach, reducing the length of hospital stay with a superimposable rate of tracheostomy decanulation time, operative time, surgical margin status, and postoperative complications. ${ }^{27}$

Table I Multidisciplinary teams (MDTs) approach in HNC

\begin{tabular}{|c|c|c|c|}
\hline Study & Methods & Diagnosis modification & Outcomes modification \\
\hline Pillay et al ${ }^{16}$ & Systematic review (72 articles) & $4 \%-45 \%$ patients & NS \\
\hline Kelly et al ${ }^{17}$ & Retrospective & $\begin{array}{l}\text { Dental evaluation }(p<0.000 \mathrm{I}) \text {, nutritional evaluation } \\
(p=0.0 \mathrm{I}) \text {, PET staging }(p<0.000 \mathrm{I}), \text { CRT in LAHNC } \\
(p<0.000 \mathrm{I}) \text { and adjuvant CRT }(p<0.000 \mathrm{I})\end{array}$ & NR \\
\hline Bergamini et al ${ }^{18}$ & Retrospective & $\begin{array}{l}\text { New staging evaluation in } 49 \% \text { of patients and therapeutic } \\
\text { changes in } 10 \%\end{array}$ & NR \\
\hline Licitra et $\mathrm{a}^{23}$ & Systematic review & $\begin{array}{l}\text { MDTs positively affect treatment decisions; reduce time to } \\
\text { treatment }\end{array}$ & Positive \\
\hline
\end{tabular}

Abbreviations: CRT, chemoradiotherapy; HNC, head and neck cancer; LAHNC, locally advanced HNC; NR, not reported; NS, not significant; PET, positron emission tomography. 
However, for transoral surgery, robust prospective trials are awaited to confirm its role in clinical practice. A challenge is the training curve and the cost of this kind of surgery that must ultimately be located in highly specialized tertiary centers.

\section{Radiotherapy}

Three-dimensional (3D) conformal RT is the standard technique used in HNSCC. ${ }^{28}$ Progress in RT led to the diffusion of 3D conformal RT or intensity-modulated RT (IMRT), image-guided RT (IGRT), and adaptive RT (ART) planning. IMRT minimizes normal organ exposure while delivering high-dose RT to a target volume. The PARSPORT trial analyzed xerostomia in 88 patients with OPC and hypopharyngeal carcinomas and showed that IMRT vs conventional RT reduced it from $75 \%$ to $39 \%$ at 1 year. ${ }^{29}$ Surgery is effective in early-stage oral cavity cancers, accessible and less sensitive to RT: OPC, hypopharynx, and larynx cancers may receive radical RT or conservative $\mathrm{S}$ with almost superimposable OS and functional outcome, while naso-pharynx cancers are treated exclusively with RT. ${ }^{28}$

Institutional experiences have also shown improvement in swallowing function and QoL measures with IMRT. ${ }^{30-33}$ In recent years, IGRT has been used not only to more precisely deliver the dose to the planned target volume (PTV) but also to allow for a reduction of margins in healthy tissues around clinical target volume (CTV) with the aim of sparing more normal tissues. Initial data show that late toxicity is less with a reduction from 5 to $3 \mathrm{~mm}$ CTV-to-PTV margins, while locoregional control is maintained. However, more data are needed to conclude whether this approach is safe and that the increased sparing of normal tissues is clinically meaningful. ${ }^{34}$

IGRT has been used also for ART, where the adjustment of treatment planning during the course of radiation accounts for anatomic changes and improvement in the therapeutic index. Few clinical studies have been published till date showing high rates of local control. ${ }^{35}$ In a single institution retrospective study, it was reported that improved local control was possible with the use of ART; however, selection biases made this conclusion questionable. ${ }^{36}$ Another study demonstrated an improvement in global QoL scales when IMRT with replanning was used, compared to those without ART, but toxicity benefits are yet to be clearly demonstrated. ART still remains labor intensive and resource demanding, and there is still a clear lack of selection criteria for patients who could better benefit from this approach. ${ }^{37}$

Several trials supported altered fractionated RT in earlystage HNSCC and demonstrated an advantage over standard
RT. In the GORTEC trial, accelerated RT improved the locoregional control rate at 6 years by $24 \% .{ }^{38}$ Recent data presented at the last ICHNO 2017 by Blanchard ${ }^{39}$ showed that, on the basis of a meta-analysis, hypofractionated RT is the best choice for early glottic cancer, while hyperfractionated RT may be superior to that of CRT after surgery. ${ }^{39}$

A worldwide IMRT technique involves the simultaneous integrated boost (SIB)-IMRT. This technique simultaneously delivers different dose levels to different target volumes in a single treatment; this shortens the treatment time and allows to increase fraction size to boost the volume. ${ }^{40,41}$

Moreover, recently, the application of volumetric intensity-modulated arc therapy (VMAT) appears to increase therapeutic action in respect to the time-consuming, highermonitor unit (MU) delivery of IMRT. ${ }^{42}$

In early-stage OPC, IMRT was compared with TORS. IMRT seems to achieve comparable or even superior oncologic and functional outcomes. However, no definitive recommendation can be made since the median follow-up was relatively short and the studies on TORS enrolled, on average, earlier-stage OPCs compared to IMRT studies. ${ }^{43,44}$

\section{Medical and RT management in LAHNC}

In LAHNC, the only $\mathrm{S}$ alone is at high risk of relapse and adjuvant therapy usually recommended. In the RTOG 73-03 trial, adjuvant RT improved loco-regional control (LRC) in respect to neoadjuvant $\mathrm{RT}^{45}$

When postoperative RT (PORT) is given without CT, the package S plus PORT should be offered (improved LRC in treatment package $<11$ weeks). The randomized trials RTOG 9501 and EORTC 22931 applied similar schedules with RT plus cisplatin and demonstrated respectively increased LRC and progression free survival (PFS). ${ }^{46,47}$ In the two trials, definition of high risk was different: in EORTC 22931, were considered as high-risk patients with the presence of a tumor at the surgical section margins (at $\leq 5 \mathrm{~mm}$ ), extracapsular extension of nodal disease, involvement of lymph nodes at levels 4 or 5 in tumors of the oral cavity or OPC, perineural disease, and/or vascular embolism. ${ }^{48}$ In the RTOG 9501 trial, high risk was defined by the presence of a tumor at the surgical section margins, extra-capsular extension of nodal disease, and/or $\geq 2$ lymph nodes involved. ${ }^{46}$ OS was significantly higher in the EORTC $22931 .{ }^{47}$

When the results of the EORTC and RTOG trials were considered in a meta-analysis, a statistically significant survival improvement $(\mathrm{HR}=0.78)$ was reported in favor of PORT. ${ }^{48}$ A more accurate selection of postoperative therapy may be done after taking into consideration many factors 
that affect tumor behavior and patient prognosis. In 2005 , Langendijk et $\mathrm{al}^{49}$ reported a recursive partitioning analysis (RPA) on HNSCCs treated with curative S and PORT. Intermediate risk (RPA I) included patients without N3 nodes, safe surgical margins ( $\geq 5 \mathrm{~mm}$ ), and the absence of extranodal spread (ENS). High-risk score (RPA II) patients had one ENS positive node or T1, T2, T3, or T4 status with close/positive margins. Very high-risk (RPA III) included patients with $\mathrm{N} 3$ neck or $\geq 2$ ENS positive nodes or T3 with close/positive margins. ${ }^{49}$

In the future, a variety of biological, pathological, and molecular factors that affect the behavior and the prognosis (e.g., tumor infiltrates lymphocyte, HPV status) might allow a more accurate selection of ENS postoperative treatment. In unresectable LAHNC, the standard treatment is cisplatinRT, with the 3-weekly schedule. ${ }^{50}$ Treatment compliance and completion rate correlate with outcomes. ${ }^{51}$

The role of induction chemotherapy (IC) is still debated. Some clinical studies reported positive results in selected patients (e.g., candidate to robotic surgery). ${ }^{52}$ Popovtzer et $\mathrm{al}^{53}$ systematically reviewed in a meta-analysis all the randomized controlled trials that assessed the addition of IC prior to CRT. Although IC produced no significant effect on OS or PFS, an advantage in complete response (CR) and disease control, as well as a trend to improved OS in the IC group, was observed. ${ }^{53}$

Neck surveillance vs dissection is another unresolved question in LAHNC multidisciplinary management, after both CRT and IC followed by surgery or CRT. The importance of node counts when neck dissection (ND) is requested has been confirmed by secondary analysis RTOG 9501 and 0234 trials. The presence of $\geq 18$ nodes was associated with better OS and higher rates of LRC, both in p16-positive and p16-negative patients. Thus, the removal of $\geq 18$ nodes might be a measure of quality in ND for mucosal squamous cell carcinoma. ${ }^{54}$

In HNSCC, fluoro-deoxy-glucose (FDG) uptake in normal tissues within the irradiated volume, measured by PET during treatment, has important prognostic value. PET computed tomography surveillance was favorably compared with ND in several trials and may be considered a standard option in several situations. ${ }^{55}$

Data obtained with immunotherapy in R/M HNSCC led to clinical trials investigating both the combination of CRT plus checkpoint inhibitors or CRT followed by checkpoint inhibitors in LAHNC. ${ }^{56}$

\section{Medical management in R/M HNC}

Patients with R/M HNC have a poor prognosis, with current systemic therapy options after failure of first-line platinum-based CT yielding an overall response rate (ORR) of $\sim 10 \%$ and an OS of 6 months. ${ }^{1}$

First-line treatment consists of platinum-based chemotherapy + the anti-epidermal growth factor receptor (EGFR) antibody $(\mathrm{Ab})$ cetuximab, followed by maintenance of cetuximab. This therapy increased the OS from 7 to 10 months in all primaries. ${ }^{57}$

Currently, no standard second-line treatment may be offered to R/M HNC. In preclinical models, vascular endothelial growth factor receptor (VEGFR), platelet-derived growth factor receptor (PDGFR), and c-kit are frequently overexpressed. For this reason, they represent a suitable target for more efficacious therapies. Several mechanisms are involved in the resistance of EGFR inhibitors: deregulation of PI3K-AKT-mTOR signaling and MET signaling, and/or upregulation of angiogenesis by VEGFR activation. However, disappointing results were reported with all targeted agents (dovitinib, axitinib, pazopanib, bevacizumab, gefitinb, and erlotinib), except afatinib. ${ }^{58}$

In the phase III LUX-Head and Neck 1 (LHN1) trial, improved PFS was observed with afatinib vs methotrexate in $\mathrm{R} / \mathrm{M} \mathrm{HNC}$ patients progressing on/after platinum-based treatment. ${ }^{59} \mathrm{~A}$ schematic flowchart is reported as suggestion of treatment both for LAHNC and R/M HNC in Figure 1.

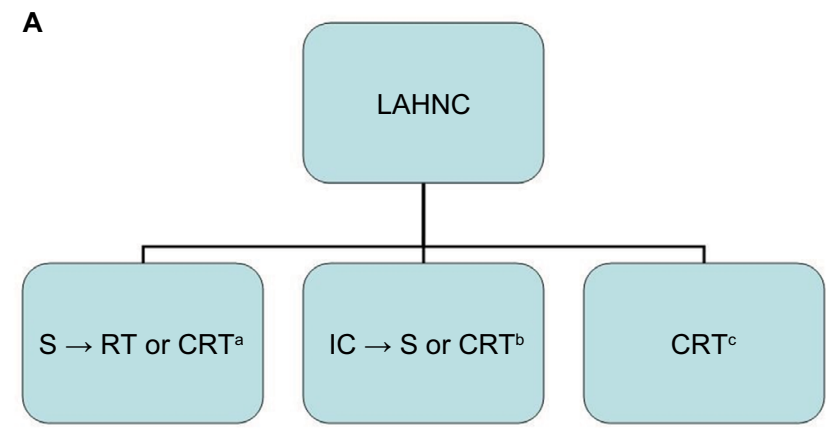

B

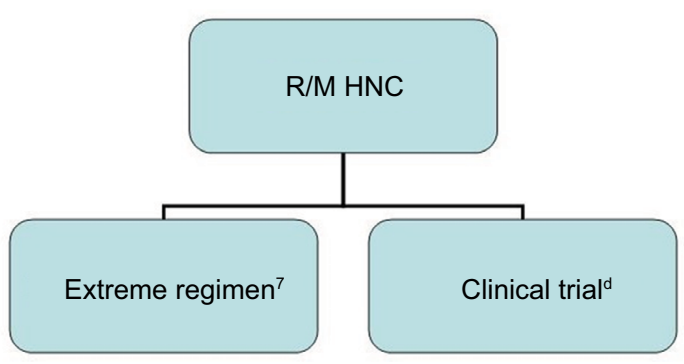

Figure I Schematic flowchart suggested for the treatment of LAHNC (A) and R/M HNC (B).

Notes: ${ }^{a}$ In patients without high-risk feature ${ }^{60}$, b preferred in organ preservation ${ }^{6}$, 'preferred in oropharyn ${ }^{5}$, ${ }^{2}$ immunotherapy is not approved nor reimbursed in Italy. Abbreviations: CRT, chemoradiotherapy; IC, induction chemotherapy; LAHNC, locally advanced head and neck cancer; R/M HNC, recurrent/metastatic head and neck cancer; RT, radiotherapy; S, surgery. 


\section{The current role of immunotherapy in HNC}

The immune system plays a key role in cancer, as tumor cells evade immune surveillance by exploiting inhibitory checkpoints that suppress antitumor $\mathrm{T}$ cell responses. ${ }^{61,62}$ The increasing understanding of the mechanisms (such as the presence of tumor-secreted proteins that act as inhibitory stimuli, cytokines, and $\mathrm{T}$ cell apoptosis) used by the immune system to control the tumor sustained the high number of novel anticancer immune-based approaches in HNSCC. ${ }^{63,64}$

Tumor progression in HPV-negative HNSCC might reflect the inability of the immune system to eliminate the tumor; even tobacco and alcohol seem stronger causative factors.

Different from other solid cancers, the presence of T-regulatory cells (Tregs) correlated with good clinical response. ${ }^{65}$ Suppression of inflammation triggered Tregs, and the elimination of immune cell with a protumor effect in cancer development and the induction of apoptosis might explain this paradox. ${ }^{66}$

The immune system plays an important also in HPVassociated OPC. Even if HPV infection is common, only a minority of subjects develop a tumor. In fact, HPV-specific effector $\mathrm{T}$ cells are responsible for elimination of the virus and HPV-induced oncogenesis correlated with weak HPVspecific $\mathrm{T}$ cell responses. On the other hand, programmed death 1 receptor (PD-1) acts as an immune checkpoint and prevent $\mathrm{T}$ cell activation. In HPV-positive OPC, PD-1 was found in tonsillar crypts and PD-1 infiltrating lymphocytes, suggesting its importance in this tumor entity. ${ }^{67}$

Moreover, HPV-induced immune-escape is complex, involving interferon (IFN) genes, microenvironment alteration, reduction of HLA class I expression, and impairing of antigen (Ag) processing machinery (APM). It has been shown that viral infection triggers the activation of IFN receptor 9 (IRF9) forming a complex (ISGF3 complex) that translocates to the nucleus and binds to specific regulatory DNA sequences interfering with the transcription of many
IFN-stimulated genes (ISGs). ${ }^{68}$ Table 2 summarizes the immunologic activity of HPV proteins.

Immunotherapy represents a further modality of multidisciplinary approach. Recent promise comes from the development of checkpoint blocking $\mathrm{Ab}$, such as those against cytotoxic T-lymphocyte antigen 4 (CTLA-4) and PD-1. They both function as negative regulators, but play a nonredundant role in immune responses. CTLA-4 negatively regulates the early activation of naive and memory T cells; PD-1 and its ligands (PD-L1 and PD-L2) are mainly involved in the modulation of $\mathrm{T}$ cell activity in peripheral tissues. ${ }^{74}$

Several anti-PD-1 and anti-PD-L1 antibody $(\mathrm{Ab})$ are under investigation, but two of them (nivolumab and pembrolizumab) were already approved by Food and Drug Administration (FDA) (respectively in August and in November 2016) in patients progressing during or after platinum-based therapy for R/M disease. ${ }^{75,76}$ Pembrolizumab is an anti-PD1 IgG4. The Keynote 012 was the first study in this setting (stage I, basket trial): 150 pretreated patients of which $37.9 \%$ had received at least three lines; the resulting response rate (RR) was $24.8 \%$, with no difference among p16-positive and p16-negative. ${ }^{75}$ In the Phase II Keynote 055 (on 172 patients), $70 \%$ had a treatment-related toxicity but only $12 \%$ experienced G3-4 adverse effects. The RR was $18 \%$ and $18 \%$ of cases showed disease stability. Final results of the Phase III study comparing pembrolizumab to a second line chosen by the investigator (including docetaxel, cetuximab, and methotrexate) will probably be presented at ASCO 2017.

Nivolumab is another anti-PD1 IgG4. In the Phase III Checkmate 141 of 361 patients enrolled, 240 received nivolumab as a second-line therapy, achieving a survival rate at 1 year by $36 \%$ vs $16 \%$ in the standard therapy (as selected by the investigator). Also, nivolumab is associated with an improvement in QoL. ${ }^{76}$

Durvalumab is an anti PD-L1 IgG1, evaluated in patients with $\mathrm{R} / \mathrm{M}$ disease after failure of platinum-based $\mathrm{CT}$ achieved ORR $12 \%$. Current studies are ongoing both in first and

Table 2 Function of HPV proteins

\begin{tabular}{ll}
\hline LI & Major capsid protein \\
L2 & Minor capsid protein \\
EI/2 & Viral replication \\
E4 & Assembly and release viral particle \\
E5 & Interaction with HLA-I heavy chain and reduced HLA-I in cell surface. ${ }^{69,70}$ Interaction with EGF \\
E7 & Downregulation of cell expression both of HLA class I, and transporter associated with antigen processing $\left(\right.$ TAP)..$^{71}$ A possible \\
& mechanism hypotheses E7 to interact with IRF-I and disrupts control of genes crucial in antigen expression. ${ }^{72}$ Inactivation of Rb \\
E6 & Inhibition of STAT-I pathway. By various mechanisms, the early viral genes alter the infected epithelial cells and prevent immune \\
& detection by antiviral T cells. ${ }^{73}$ Destruction of p53
\end{tabular}

Abbreviations: EGF, epidermal growth factor; HLA-I, human leukocyte antigen class I; IRF-I, interferon regulatory factor I; Rb, retinoblastoma; STAT-I, signal transducer and activator of transcription I. 
second line investigating this drug in monotherapy or in combination with anti-CTLA-4 inhibitor tremelimumab. ${ }^{77}$

Despite the optimism for the introduction of immune checkpoint inhibitors, we must consider that more than $40 \%$ of patients in studies Keynote $012,{ }^{75}$ Keynote $055,{ }^{78}$ and Checkmate $141^{76}$ progressed during treatment.

Several combinations with immune checkpoint agonists (such as urelumab-CD137 and lirilumab-Kir2) are ongoing with very interesting preliminary results. ${ }^{77}$

Response evaluation with immunotherapy may be difficult because of lymphocyte tumor infiltration (TIL) that is associated with response (pseudo-progression). Pseudoprogression in HNSCC is less common than in melanoma; although continuation until response confirmation is difficult in this setting, because it is often (especially in the oral cavity patients) symptomatic. However, even when nivolumab or pembrolizumab therapy is suspended, some late responses were observed at $2-3$ months. ${ }^{79}$

\section{Conclusion}

Treatments of HNSCC are rapidly moving after nearly two decades of unchanging unmet needs. More recently new prognostic and predictive factors were identified, including biomolecular aspects and immune microenvironment. Integration of these new factors with the old deserves dedicated clinical studies, but the available knowledge already allows us to make some deductive hypotheses.

Early-stage disease treatment is unchanged, deserving a single modality treatment. LAHNC deserves an MDT to allow better staging and treatment. Integrating chemotherapy, radiotherapy, biotherapy, and surgery is the standard treatment in this setting. Treatment choice will be based on the clinical condition of the patient, the physician's experience, and the patient's preference. Whatever is the treatment of choice, it is important to involve multidisciplinary staff in the management of these patients. Indeed, also unresectable patients may require supportive surgical interventions before or during treatment, or the removal of residual disease after treatment. $^{80}$

Immunotherapy is under investigation, but it is not a choice out of clinical trials. Adequate supportive care managed by MDT is highly recommended.

$\mathrm{R} / \mathrm{M}$ disease will benefit from immune checkpoint inhibitors following progression on platinum therapy. In the near future, the use of biomarkers predictive for immune response and the results of first-line therapy will allow the selection of patients who might benefit from upfront immunotherapy.

\section{Disclosure}

The authors report no conflicts of interest in this work.

\section{References}

1. National Comprehensive Cancer Network: Clinical Practice Guidelines in Oncology. Head and Neck Cancer v1; 2017. Available from: https:// www.nccn.org/professionals/physician_gls/f_guidelines.asp\#site.

2. Weinberger PM, Yu Z, Haffty BG, et al. Molecular classification identifies a subset of human papillomavirus-associated oropharyngeal cancers with favorable prognosis. J Clin Oncol. 2006;24(5):736-747.

3. Baxi S, Fury M, Ganly I, Rao S, Pfister DG. Ten years of progress in head and neck cancers. $J$ Natl Compr Canc Netw. 2012;10(7): 806-810.

4. Maxwell JH, Grandis JR, Ferris R. HPV-associated head and neck cancer: unique features of epidemiology and clinical management. Annu Rev Med. 2016;67:91-101.

5. Adelstein DJ, Li Y, Adams GL, et al. An intergroup phase III comparison of standard radiation therapy and two schedules of concurrent chemoradiotherapy in patients with unresectable squamous cell head and neck cancer. J Clin Oncol. 2003;21(1):92-98.

6. Forastiere AA, Zhang Q, Weber RS, et al. Long-term results of RTOG 91-11: a comparison of three nonsurgical treatment strategies to preserve the larynx in patients with locally advanced larynx cancer. J Clin Oncol. 2013;31(7):845-852.

7. Bonner JA, Harari PM, Giralt J, et al. Radiotherapy plus cetuximab for locoregionally advanced head and neck cancer: 5 -year survival data from a phase 3 randomised trial, and relation between cetuximab-induced rash and survival. Lancet Oncol. 2010;11(1):21-28.

8. Cocks H, Ah-See K, Capel M, Taylor P. Palliative and supportive care in head and neck cancer: United Kingdom National Multidisciplinary Guidelines. J Laryngol Otol. 2016;130(S2):S198-S207.

9. Bossi P, Alfieri S. Investigational drugs for head and neck cancer. Expert Opin Investig Drugs. 2016;25(7):797-810.

10. Badoual C, Hans S, Merillon N, et al. PD-1-expressing tumor-infiltrating $\mathrm{T}$ cells area favorable prognostic biomarker in HPV-associated head and neck cancer. Cancer Res. 2013;73(1):128-138.

11. Holsinger FC, Ferris RL. Transoral endoscopic head and neck surgery and its role within the multidisciplinary treatment paradigm of oropharynx cancer: robotics, lasers, and clinical trials. J Clin Oncol. 2015;33(29):3285-3292.

12. Schindler A, Denaro N, Russi EG, et al. Dysphagia in head and neck cancer patients treated with radiotherapy and systemic therapies: Literature review and consensus. Am J Otolaryngol. 2013;34(1):57-60.

13. Mirabile A, Airoldi M, Ripamonti C, et al. Pain management in head and neck cancer patients undergoing chemo-radiotherapy: Clinical practical recommendations. Crit Rev Oncol Hematol. 2016;99:100-106.

14. De Sanctis V, Bossi P, Sanguineti G, et al. Mucositis in head and neck cancer patients treated with radiotherapy and systemic therapies: Literature review and consensus statements. Crit Rev Oncol Hematol. 2016;100:147-166.

15. Buglione M, Cavagnini R, Di Rosario F, et al. Oral toxicity management in head and neck cancer patients treated with chemotherapy and radiation: Xerostomia and trismus (Part 2). Literature review and consensus statement. Crit Rev Oncol Hematol. 2016;102:47-54.

16. Pillay B, Wootten AC, Crowe $\mathrm{H}$, et al. The impact of multidisciplinary team meetings on patient assessment, management and outcomes in oncology settings: a systematic review of the literature. Cancer Treat Rev. 2016;42:56-72.

17. Kelly SL, Jackson JE, Hickey BE, Szallasi FG, Bond CA. Multidisciplinary clinic care improves adherence to best practice in head and neck cancer. Am J Otolaryngol. 2013;34(1):57-60.

18. Bergamini C, Locati L, Bossi P, et al. Does a multidisciplinary team approach in a tertiary referral centre impact on the initial management of head and neck cancer? Oral Oncol. 2016;54:54-57. 
19. Birchall M, Bailey D, King P; South West Cancer Intelligence Service Head and Neck Tumor Panel. Effect of process standards on survival of patients with head and neck cancer in the south and west of England. Br J Cancer. 2004;91(8):1477-1481.

20. Friedland PL, Bozic B, Dewar J, Kuan R, Meyer C, Phillips M. Impact of multidisciplinary team management in head and neck cancer patients. Br J Cancer. 2011;104(8):1246-1248.

21. Wang YH, Kung PT, Tsai WC, Tai CJ, Liu SA, Tsai MH. Effects of multidisciplinary care on the survival of patients with oral cavity cancer in Taiwan. Oral Oncol. 2012;48(9):803-810.

22. Patil RD, Meinzen-Derr JK, Hendricks BL, Patil YJ. Improving access and timeliness of care for veterans with head and neck squamous cell carcinoma: a multidisciplinary team's approach. Laryngoscope. 2016;126(3):627-631.

23. Licitra L, Keilholz U, Tahara M, et al. Evaluation of the benefit and use of multidisciplinary teams in the treatment of head and neck cancer. Oral Oncol. 2016;59:73-79.

24. Wuthrick EJ, Zhang Q, Machtay M, et al. Institutional clinical trial accrual volume and survival of patients with head and neck cancer. J Clin Oncol. 2015;33(2):156-164.

25. Steiner W. Experience in endoscopic laser surgery of malignant tumors of the upper aerodigestive tract. Adv Otorhinolaryngol. 1988;39:135-144

26. Yee S. Transoral robotic surgery. AORN J. 2017;105(1):73-84.

27. Biron VL, O'Connell DA, Barber B, et al. Transoral robotic surgery with radial forearm free flap reconstruction: case control analysis. J Otolaryngol Head Neck Surg. 2017;46(1):20.

28. Corvò R. Evidence-based radiation oncology in head and neck squamous cell carcinoma. Radiother Oncol. 2007;85(1):156-170.

29. Nutting CM, Morden JP, Harrington KJ, et al; PARSPORT trial management group. Parotid-sparing intensity modulated vs conventional radiotherapy in head and neck cancer (PARSPORT): a phase 3 multicentre randomised controlled trial. Lancet Oncol. 2011;12(2):127-136.

30. Kotz T, Costello R, Li Y, Posner MR. Swallowing dysfunction after chemoradiation for advanced squamous cell carcinoma of the head and neck. Head Neck. 2004;26(4):365-372.

31. Feng FY, Kim HM, Lyden TH, et al. Intensity-modulated chemoradiotherapy aiming to reduce dysphagia in patients with oropharyngeal cancer: clinical and functional results. J Clin Oncol. 2010;28(16):2732-2738.

32. Eisbruch A, Kim HM, Feng FY, et al. Chemo-IMRT of oropharyngeal cancer aiming to reduce dysphagia: Swallowing organs late complication probabilities and dosimetric correlates. Int J Radiat Oncol Biol Phys. 2011;81(3):e93-e99.

33. Kraaijenga SC, Oskam IM, van der Molen L, Hamming-Vrieze O, Hilgers FJM, van den Brekel MW. Evaluation of long term (10-years +$)$ dysphagia and trismus in patients treated with concurrent chemo-radiotherapy for advanced head and neck cancer. Oral Oncol. 2015;51(8):787-794.

34. Chen AM, Yu Y, Daly ME, Farwell DG, Benedict SH, Purdy JA. Longterm experience with reduced planning target volume margins and intensity-modulated radiotherapy with daily image-guidance for head and neck cancer. Head Neck. 2014;36(12):1766-1772.

35. Schwartz DL, Garden AS, Shah SJ, et al. Adaptive radiotherapy for head and neck cancer--dosimetric results from a prospective clinical trial. Radiother Oncol. 2013;106(1):80-84.

36. Chen AM, Daly ME, Cui J, Mathai M, Benedict S, Purdy JA. Clinical outcomes among patients with head and neck cancer treated by intensitymodulated radiotherapy with and without adaptive replanning. Head Neck. 2014;36(11):1541-1546.

37. Yang H, Hu W, Wang W, Chen P, Ding W, Luo W. Replanning during intensity modulated radiation therapy improved quality of life in patients with nasopharyngeal carcinoma. Int J Radiat Oncol Biol Phys. 2013;85(1):e47-e54.

38. Bourhis J, Lapeyre M, Tortochaux J, et al. Phase III randomized trial of very accelerated radiation therapy compared with conventional radiation therapy in squamous cell head and neck cancer: a GORTEC trial. J Clin Oncol. 2006;24(18):2873-2878
39. Blanchard P. 6th ICHNO: International Conference on Innovative Approaches in Head \& Neck Oncology; 16th-18th March 2017. Barcellona, Spain.

40. Orlandi E, Palazzi M, Pignoli E, Fallai C, Giostra A, Olmi P. Radiobiological basis and clinical results of the simultaneous integrated boost (SIB) in intensity modulated radiotherapy (IMRT) for head and neck cancer: a review. Crit Rev Oncol Hematol. 2010;73(2):111-125.

41. Gutiontov SI, Shin EJ, Lok B, Lee NY, Cabanillas R. Intensity-modulated radiotherapy for head and neck surgeons. Head Neck. 2016;38 (Suppl 1):E2368-E2373.

42. Osborn J. Is VMAT beneficial for patients undergoing radiotherapy to the head and neck? Radiography (Lond). 2017;23(1):73-76.

43. Yeh DH, Tam S, Fung K, MacNeil SD, et al. Transoral robotic surgery vs radiotherapy for management of oropharyngeal squamous cell carcinoma - A systematic review of the literature. Eur J Surg Oncol. 2015;41(12):1603-1614.

44. Veresezan O, Troussier I, Lacout A, et al. Adaptive radiation therapy in head and neck cancer for clinical practice: state of the art and practical challenges. Jpn J Radiol. 2017;35(2):43-52.

45. Tupchong L, Scott CB, Blitzer PH, et al. Randomized study of preoperative vs postoperative radiation therapy in advanced head and neck carcinoma: long-term follow-up of RTOG study 73-03. Int $J$ Radiat Oncol Biol Phys. 1991;20(1):21-28.

46. Cooper JS, Pajak TF, Forastiere AA, et al; Radiation Therapy Oncology Group 9501/Intergroup. Postoperative concurrent radiotherapy and chemotherapy for high-risk squamous-cell carcinoma of the head and neck. N Engl J Med. 2004;350(19):1937-1944.

47. Bernier J, Domenge C, Ozsahin M, et al; European Organization for Research and Treatment of Cancer Trial 22931. Postoperative irradiation with or without concomitant chemotherapy for locally advanced head and neck cancer. N Engl J Med. 2004;350(19):1945-1952.

48. Bernier J, Cooper JS, Pajak TF, et al. Defining risk levels in locally advanced head and neck cancers: a comparative analysis of concurrent postoperative radiation plus chemotherapy trials of the EORTC (\#22,931) and RTOG (\# 9501). Head Neck. 2005;27(10):843-850.

49. Langendijk JA, Slotman BJ, van der Waal I, Doornaert P, Berkof J, Leemans CR. Risk-group definition by recursive partitioning analysis of patients with squamous cell head and neck carcinoma treated with surgery and postoperative radiotherapy. Cancer. 2005;104(7):1408-1417.

50. Pignon JP, le Maître A, Maillard E, Bourhis J; MACH-NC Collaborative Group. Meta-analysis of chemotherapy in head and neck cancer (MACH-NC): an update on 93 randomised trials and 17346 patients. Radiother Oncol. 2009;92(1):4-14.

51. Salama JK, Mell LK, Schomas DA, et al. Concurrent chemotherapy and intensity-modulated radiation therapy for anal canal cancer patients: a multicenter experience. J Clin Oncol. 2007;25(29):4581-4586.

52. Cossu Rocca M, Maffini F, Chiocca S, et al. Induction chemotherapy followed by transoral laser microsurgery: a multimodal approach to improve outcomes for locally advanced laryngeal cancer patients? J Clin Oncol. 2015;33 (Suppl 15): abstr e17039.

53. Popovtzer A, Burnstein H, Stemmer S, et al. Phase II organ-preservation trial: Concurrent cisplatin and radiotherapy for advanced laryngeal cancer after response to docetaxel, cisplatin, and 5-fluorouracil-based induction chemotherapy. Head Neck. 2017;39(2):227-233.

54. Divi V, Harris J, Harari PM, et al. Establishing quality indicators for neck dissection: Correlating the number of lymph nodes with oncologic outcomes (NRG Oncology RTOG 9501 and RTOG 0234). Cancer. Epub 2016 Jul 15.

55. Zschaeck S, Löck S, Leger S, et al. FDG uptake in normal tissues assessed by PET during treatment has prognostic value for treatment results in head and neck squamous cell carcinomas undergoing radiochemotherapy. Radiother Oncol. 2017;122(3):437-444.

56. Mandal R, Şenbabaoğlu Y, Desrichard A, et al. The head and neck cancer immune landscape and its immunotherapeutic implications. JCI Insight. 2016;1(17):e89829.

57. Vermorken JB, Mesia R, Rivera F, et al. Platinum-based chemotherapy plus cetuximab in head and neck cancer. $N$ Engl $J$ Med. 2008;359(11):1116-1127. 
58. Specenier P, Vermorken J. Afatinib in squamous cell carcinoma of the head and neck. Expert Opin Pharmacother. 2016;17(9):1295-1301.

59. Machiels JP, Haddad RI, Fayette J, et al; LUX-H\&N 1 investigators. Afatinib versus methotrexate as second-line treatment in patients with recurrent or metastatic squamous-cell carcinoma of the head and neck progressing on or after platinum-based therapy (LUX-Head \& Neck 1): an open-label, randomised phase 3 trial. Lancet Oncol. 2015;16(5):583-594.

60. Denaro N, Russi EG, Adamo V, Colantonio I, Merlano MC. Postoperative therapy in head and neck cancer: state of the art, risk subset, prognosis and unsolved questions. Oncology. 2011;81(1):21-29.

61. Mellman I, Coukos G, Dranoff G. Cancer immunotherapy comes of age. Nature. 2011;480(7378):480-489.

62. Economopoulou P, Perisanidis C, Giotakis EI, Psyrri A. The emerging role of immunotherapy in head and neck squamous cell carcinoma (HNSCC): anti-tumor immunity and clinical applications. Ann Transl Med. 2016;4(9):173.

63. Pai SI, Zandberg DP, Strome SE. The role of antagonists of the PD-1: PD-L1/PD-L2 axis in head and neck cancer treatment. Oral Oncol. 2016;61:152-158.

64. Balermpas P, Rodel F, Rodel C, et al. CD8+ tumor-infiltrating lymphocytes in relation to HPV status and clinical outcome in patients with head and neck cancer after postoperative chemoradiotherapy: a multicentre study of the German cancer consortium radiation oncology group (DKTK-ROG). Int J Cancer. 2016;138(1):171-181.

65. Ferris RL, Whiteside TL, Ferrone S. Immune escape associated with functional defects in antigen-processing machinery in head and neck cancer. Clin Cancer Res. 2006;12(13):3890-3895.

66. Ferris RL. Immunology and immunotherapy of head and neck cancer. J Clin Oncol. 2015;33(29):3293-3304.

67. Chakravarthy A, Henderson S, Thirdborough SM, et al. Human papillomavirus drives tumor development throughout the head and neck: improved prognosis is associated with an immune response largely restricted to the oropharynx. J Clin Oncol. 2016;34(34):4132-4141.

68. Padilla-Quirarte HO, Trejo-Moreno C, Fierros-Zarate G, et al. Interferontau has antiproliferative effects, represses the expression of E6 and E7 oncogenes, induces apoptosis in cell lines transformed with HPV16 and inhibits tumor growth in vivo. $J$ Cancer. 2016;7(15):2231-2240.
69. Campo MS, Graham SV, Cortese MS, et al. HPV-16 E5 down-regulates expression of surface HLA class I and reduces recognition by CD8 T cells. Virology. 2010;407(1):137-142.

70. Heusinkveld M, Goedemans R, Briet RJ, et al. Systemic and local human papillomavirus 16-specific T-cell immunity in patients with head and neck cancer. Int J Cancer. 2012;131(2):E74-E85.

71. Li W, Deng XM, Wang CX, et al. Down-regulation of HLA class I antigen in human papillomavirus type $16 \mathrm{E} 7$ expressing $\mathrm{HaCaT}$ cells: correlate with TAP-1 expression. Int J Gynecol Cancer. 2010;20(2): $227-232$.

72. Um SJ, Lee SY, Kim EJ, Myoung J, Namkoong SE, Park JS. Downregulation of human papillomavirus E6/E7 oncogene by arsenic trioxide in cervical carcinoma cells. Cancer Lett. 2002;181(1):11-22.

73. Stanley M. HPV-immune response to infection and vaccination. Infect Agent Cancer. 2010;5:19.

74. Chen DS, Mellman I. Oncology meets immunology: the cancerimmunity cycle. Immunity. 2013;39(1):1-10.

75. Seiwert TY, Burtness B, Mehra R, et al. Safety and clinical activity of pembrolizumab for treatment of recurrent or metastatic squamous cell carcinoma of the head and neck (KEYNOTE-012): an open-label, multicentre, phase 1b trial. Lancet Oncol. 2016;17(7):956-965.

76. Ferris RL, Blumenschein G Jr, Fayette J, et al. Nivolumab for recurrent squamous-cell carcinoma of the head and neck. $N$ Engl $J$ Med. 2016;375(19):1856-1867.

77. Segal NH. A phase I dose escalation and cohort expansion study of lirilumab (anti-KIR; BMS-986015) in combination with nivolumab (anti-PD-1; BMS-936558, ONO-4538) in advanced solid tumors. Meeting Library "Abstracts" 2014 ASCO Annual Meeting; Chicago, 30 May - 3 June.

78. Bauml J, Seiwert TY, Pfister DG, et al. Pembrolizumab for Platinumand Cetuximab-Refractory Head and Neck Cancer: Results From a Single-Arm, Phase II Study. J Clin Oncol. 2017;35(14):1542-1549.

79. Baxi SS, Dunn LA, Burtness BA. Amidst the excitement: a cautionary tale of immunotherapy, pseudoprogression and head and neck squamous cell carcinoma. Oral Oncol. 2016;62:147-148.

80. Denaro N, Russi EG, Merlano MC. Strategies for non-resectable head and neck cancer. Curr Treat Options Oncol. 2013;14(4):492-504.
Cancer Management and Research

\section{Publish your work in this journal}

Cancer Management and Research is an international, peer-reviewed open access journal focusing on cancer research and the optimal use of preventative and integrated treatment interventions to achieve improved outcomes, enhanced survival and quality of life for the cancer patient. The manuscript management system is completely online and includes

\section{Dovepress}

a very quick and fair peer-review system, which is all easy to use. Visit http://www.dovepress.com/testimonials.php to read real quotes from published authors. 